Check for updates

Cite this: RSC Adv., 2018, 8, 19024

\title{
Binding $\mathrm{TiO}_{2}$ nanoparticles to forward osmosis membranes via MEMO-PMMA-Br monomer chains for enhanced filtration and antifouling performance $\dagger$
}

\author{
Wenchao Xue, (D) *ab Kaung Ko Ko Sint, ${ }^{a}$ Chavalit Ratanatamskul, ${ }^{\text {*ae }}$ \\ Piyasan Praserthdam ${ }^{c}$ and Kazuo Yamamoto ${ }^{\text {bd }}$
}

Forward osmosis (FO) has attracted increasing interest in various applications for water and wastewater treatment and reuse. However, drawbacks caused by its lower-than-expected flux performance and fouling issues remain bottlenecks that limit the wider applications of FO technology. In this research, titanium dioxide $\left(\mathrm{TiO}_{2}\right)$ nanoparticles were grafted onto two commercially available $\mathrm{FO}$ membranes, a cellulose triacetate (CTA) membrane and an aquaporin (AqP) membrane, through a specially designed 3-(trimethoxysilyl)propyl methacrylate-polymethyl methacrylate-bromide (MEMO-PMMA-Br) monomer chain, to improve the filtration performance with regard to pure water flux and organic fouling resistance. The success of the surface coating method was verified using FT-IR, SEM-EDX, and AFM. Approximately $30 \%$ titanium coverage was obtained for both $\mathrm{FO}$ membranes. A reduction of the contact angle on the modified CTA membrane surface indicated enhanced water permeability and antifouling performance. An adverse effect on the surface hydrophilicity of the modified AqP membrane may be attributed to the obstruction of aquaporins from the feed solution due to the coverage of MEMOPMMA-Br monomers and $\mathrm{TiO}_{2}$ nanoparticles. The pure water flux of both membranes was significantly improved, with average flux increases of $73.4 \%$ and $13.6 \%$ identified for the modified CTA and AqP membranes, respectively. In addition, the antifouling performance of the AqP membrane was greatly enhanced after surface modification, attributed to the integrated effects of foulant photodegradation (catalyzed by $\mathrm{TiO}_{2}$ nanoparticles at the interface) and the prevention of functional water channels being blocked by organic foulants due to $\mathrm{TiO}_{2}$ coverage.

Received 26th April 2018 Accepted 17th May 2018 DOI: $10.1039 / c 8 r a 03613 f$ rsc.li/rsc-advances

\section{Introduction}

Forward osmosis (FO) technology has been highlighted as a sustainable water and wastewater treatment solution surpassing conventional pressurized membrane processes by offering advantages including: (i) low energy costs and

${ }^{a}$ Department of Environmental Engineering, Faculty of Engineering, Chulalongkorn University, 254 Phayathai Road, Patumwan, Bangkok 10330, Thailand. E-mail: dr_chawalit@yahoo.com; Fax: +66(0)2-2186678; Tel: +66(0)2-2186678

${ }^{b}$ Department of Energy, Environment, and Climate Change, School of Environment, Resources, and Development, Asian Institute of Technology, Pathumthani 12120, Thailand. E-mail: wenchao@ait.ac.th; Fax: +66(0)2-5245625; Tel: +66(0)2-5245626

${ }^{c}$ Department of Chemical Engineering, Faculty of Engineering, Chulalongkorn University, 254 Phayathai Road, Patumwan, Bangkok 10330, Thailand

${ }^{d}$ Environmental Science Center, The University of Tokyo, 7-3-1 Hongo, Bunkyo-ku, Tokyo 113-0033, Japan

${ }^{e}$ Research Unit on Innovative Waste Treatment and Water Reuse, Faculty of Engineering, Chulalongkorn University, 254 Phayathai Road, Patumwan, Bangkok 10330, Thailand

$\dagger$ Electronic supplementary information (ESI) available. See DOI: 10.1039/c8ra03613f equipment investment; (ii) high recovery of pure water due to little impact of membrane scaling; (iii) high retention of various contaminants; and (iv) relatively low membrane fouling propensity. ${ }^{1-3}$ Hence, in the last decade, the amount of scientific research and the number of reported practical applications have grown rapidly. For instance, attempts have been made to utilize FO in desalinating seawater and other saline water, ${ }^{4,5}$ producing commercial materials such as anthocyanin, ${ }^{6}$ treating and reclaiming municipal and industrial wastewater, ${ }^{7,8}$ and harvesting nutrients from waste and wastewater. ${ }^{9-11}$

Despite its great potential, the lower-than-expected flux in FO has become a bottleneck, limiting widespread utilization of this promising technology. ${ }^{12,13}$ Various solutions, including the selection of draw solutions with high osmotic potential and the employment of pressure assisted FO, have been proposed to enhance the flux performance. ${ }^{14-17}$ Nevertheless, these strategies require greater energy consumption to boost the process performance.

On the other hand, fouling is an inevitable issue for all membrane processes. The formation of membrane fouling 
which deteriorates flux performance and increases energy costs lays a major obstacle for the sustainable application of membrane technologies. Although membrane fouling is considered to be comparatively lighter in FO than that in pressurized membrane processes, ${ }^{3,18,19}$ sufficient approaches have been suggested to control and retard the development of fouling in FO such as pre-treatment of feed solution, hydraulic and chemical cleaning of membranes, and optimization of operating conditions and module configuration. ${ }^{11,20,21}$

Surface modification of the membrane material provides an alternative solution to improve the performance of membrane processes, and has been widely studied and reported for pressurized membrane processes. ${ }^{22,23}$ In particular, membrane surface modification using photocatalyst introduces an interface with advanced oxidizing properties between the feed solution and membrane. Among the various optional photocatalysts, titanium dioxide $\left(\mathrm{TiO}_{2}\right)$ is one of the most widely utilized because it is commercially available, inexpensive, chemically stable and non-toxic. ${ }^{24-26}$ Due to the presence of the hydroxyl radicals and superoxide reactive radicals which are produced by the photocatalytic irradiation of $\mathrm{TiO}_{2}$, the modified membranes are designed to provide excellent performance incorporating physical separation, advanced oxidation of organic pollutants, and inactivation of microorganisms, bacteria, and viruses. ${ }^{27}$ Furthermore, when modified by $\mathrm{TiO}_{2}$, the membrane's surface morphology and properties may induce improved water permeability and fouling resistance. Nevertheless, a major concern with $\mathrm{TiO}_{2}$ modified membranes falls on the potential degradation of polymer membrane materials through oxidation and long-time exposure to ultraviolet (UV) light during the following applications. ${ }^{28}$

Several researchers have attempted to coat $\mathrm{TiO}_{2}$ nanoparticles onto FO membranes and studied the corresponding effects brought by the modification. Nguyen et al. ${ }^{29}$ prepared a thin film composite (TFC) membrane with surface modification in the form of silver/ $/ \mathrm{TiO}_{2}$ nanoparticles, deposited via either the conventional sol-gel method or charge-driven selfassembly. The modified membrane effectively inhibited biofilm growth, although, this was primarily attributed to the antibacterial effect of silver nanoparticles. The role of $\mathrm{TiO}_{2}$ in this study was concluded to "regenerate" the silver particles that were covered by organic matter. Huang et al. ${ }^{30}$ attempted to assemble $\mathrm{TiO}_{2}$ nanoparticles onto TFC membranes via polydopamine. Nevertheless, their major focus was to remove pharmaceuticals using the modified membrane. The effects the of $\mathrm{TiO}_{2}$ coating on membrane fouling resistance and membrane integrity were not evaluated.

In the current work, we attempted to graft $\mathrm{TiO}_{2}$ nanoparticles onto the active layers of two commercially available FO membranes, a cellulose triacetate (CTA) membrane and an aquaporin (AqP) membrane, via a 3-(trimethoxysilyl)propyl methacrylate-polymethyl methacrylate-bromide (MEMOPMMA-Br) monomer chain. The aim was to improve membrane performance in terms of water permeability, salt rejection, and organic fouling resistance. The monomer chain was designed to play as an intermedia between the $\mathrm{TiO}_{2}$ nanoparticles and the FO membranes, to protect the active polymer surfaces from degradation caused by the deposition of $\mathrm{TiO}_{2}$. The surface morphology, chemical components, and properties were then evaluated to confirm the success of the $\mathrm{TiO}_{2}$ coating and effects brought by surface modification. Improvement of water permeability and antifouling performance was examined in a laboratory-scale FO filtration system with a light-emitting diode (LED) light applied instead of UV irradiation to further reduce the effect on the polymer membranes.

\section{Materials and methods}

\subsection{FO membranes}

Two commercial FO membranes were modified and studied. The first is the CTA membrane provided by Hydration Technology Innovations (Albany, OR, USA), the performance and properties of which have been extensively investigated and reported in previous literatures. ${ }^{11,31,32}$ The CTA membrane is composed of a cellulose triacetate active layer with an embedded woven support mesh. ${ }^{33}$ The second is the AqP membrane which was procured from Aquaporin A/S (Copenhagen, Denmark). The AqP membrane is manufactured by embedding biomimetic aquaporin protein channels into a polyamide active layer. ${ }^{34,35}$ The membrane sheets were soaked in pure water (Millipore, USA) for 24 hours to remove residual preservative reagents prior to the filtration experiments.

\subsection{FO membrane surface modification}

The MEMO-PMMA-Br monomer chain was prepared following the procedure described by Klaysri et al. ${ }^{36}$ The MEMO-PMMA$\mathrm{Br}$ gel was initially diluted using tetrahydrofuran (THF, Unilab) at a volume ratio of $1: 1$. The diluted MEMO-PMMA-Br reagent was then uniformly sprayed over the active surfaces of FO membranes. After drying for $30 \mathrm{~min}$ at room temperature, the active surface was dipped into a sulfuric acid solution $\left(\mathrm{H}_{2} \mathrm{SO}_{4}\right.$, Qrec) at $\mathrm{pH} 3$ to hydrogenate the monomer molecules for $30 \mathrm{~min}$. The hydrogenated MEMO-PMMA-Br chain contains three hydroxyls on the silyl, which are able to form strong bindings with $\mathrm{TiO}_{2}$ nanoparticles through the reactions of silanization. ${ }^{36,37}$

A suspension of $\mathrm{TiO}_{2}$ nanoparticles was prepared using commercial $\mathrm{TiO}_{2}$ nanopowder (AEROXIDE®). The nanopowder was dissolved in ethanol $\left(\mathrm{C}_{2} \mathrm{H}_{5} \mathrm{OH}\right.$, Merck) with $\mathrm{pH}$ adjusted to 10 by ammonia hydroxide $\left(\mathrm{NH}_{4} \mathrm{OH}\right.$, Sigma-Aldrich). After agitating for $1 \mathrm{~h}$ at $1000 \mathrm{rpm}$, the mixture was further treated using ultrasonic for $15 \mathrm{~min}$ in a water bath at $25{ }^{\circ} \mathrm{C}$ to obtain a well dispersed $\mathrm{TiO}_{2}$ nanoparticle suspension. The pretreated FO membrane was subsequently immersed in the $\mathrm{TiO}_{2}$ nanoparticle suspension for $30 \mathrm{~min}$. After three cycles of ultrasonic and dipping treatment, the membrane was rinsed using ethanol and stored in distilled water at $4{ }^{\circ} \mathrm{C}$ until further examination. The membrane coating procedures are illustrated in Fig. 1.

\subsection{Membrane surface characterization}

The major functional groups on the membrane surfaces before and after modification were identified using Fourier transform infrared spectroscopy (FT-IR, Nicolet 6700, Thermo Electron 


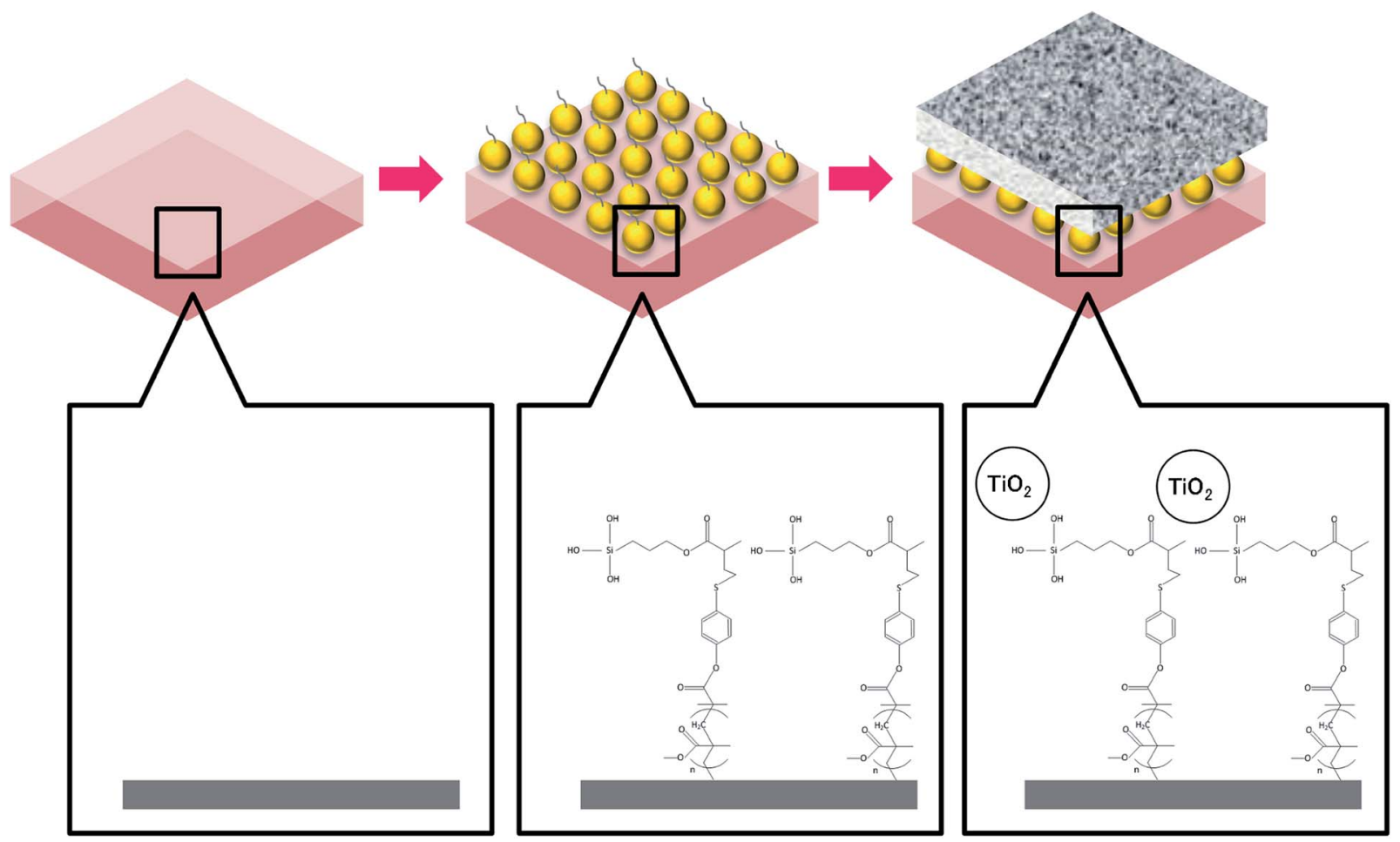

Fig. 1 Procedures of membrane surface modification by $\mathrm{TiO}_{2}$ nanoparticles via MEMO-PMMA-Br monomer chains.

CO., USA). The FT-IR spectra for each membrane sample were collected from wave numbers between 500 and $4000 \mathrm{~cm}^{-1}$ at a resolution of $2 \mathrm{~cm}^{-1}$.

The surface morphology and chemical composition of the virgin and modified membranes were assessed using scanning electron microscopy (SEM, S-3400N, Hitachi, Japan) in conjunction with energy dispersive X-ray spectroscopy (EDX, Apollo X, EDAX, USA). Scans were obtained with an electron accelerating voltage of $15 \mathrm{kV}$, a tilt angle of the specimen stage of $0^{\circ}$, a working distance of $10 \mathrm{~mm}$ and vacuum degree of $30 \mathrm{~Pa}$. The membrane surface was further observed using atomic force microscopy (AFM, MMAFMLN, Veeco Instruments Inc., USA) to visualize the surface roughness before and after the coating procedure. Three-dimensional images were obtained by scanning a surface area of $30 \times 30 \mu \mathrm{m}$ in non-contact mode with a scan rate of $0.5 \mathrm{~Hz}$. In addition, two-dimensional surface profile measurements were conducted using a contact stylus profilometer (Dektak 150, Veeco Instruments Inc., USA) to support the results obtained by AFM.

The hydrophilicity of membrane active surfaces was evaluated by the water contact angle using a contact angle analyzer (CAM-PLUS IMAGE, C1221105, Tantec Inc., USA). The measurements were performed at three random locations for each membrane sample with the average values reported in this study.

\subsection{Laboratory-scale FO setup and operation}

The laboratory-scale FO (Fig. S1 $\dagger$ ) was modified based on the bench top system that was adopted in our previous work. ${ }^{33}$
Counter-current circulation of draw solution and feed solution was controlled via peristaltic pumps (BT100-2J, LongerPump, China). A cross-flow velocity of $8.3 \mathrm{~cm} \mathrm{~s}^{-1}$ was set at each side of the membrane for all filtration tests. A 5 watt LED light was employed to irradiate the $\mathrm{TiO}_{2}$ nanoparticles during the filtrations. All experiments were run at room temperature (approximately $25{ }^{\circ} \mathrm{C}$ ). Water flux of $\mathrm{FO}$ was determined by measuring the rate at which the weight of the feed solution decreased using an electronic balance (UW4200H, Shimadzu, Japan), by:

$$
J_{\mathrm{W}}=\frac{\Delta V}{A \Delta t}
$$

where $J_{\mathrm{W}}$ is the water flux of FO, $\mathrm{L} \mathrm{m}^{-2} \mathrm{~h}^{-1} ; \Delta V$ is the permeate volume over a predetermined time interval, $\mathrm{L} ; \Delta t$ is the predetermined time internal, $h$; and $A$ is the effective filtration area of the membrane, $\mathrm{m}^{2}$.

The reverse solute diffusion was determined via monitoring the conductivity variation in the feed solution. The reverse solute flux was calculated as:

$$
J_{\mathrm{DS}}=\frac{c_{\mathrm{f}, t} V_{t}-c_{\mathrm{f}, 0} V_{0}}{A \Delta t}
$$

where $J_{\mathrm{DS}}$ is the reverse solute flux of FO, mmoL m ${ }^{-2} \mathrm{~h}^{-1} ; c_{\mathrm{f}, t}$ and $c_{\mathrm{f}, 0}$ are the draw solute concentrations in the feed solution, $\mathrm{mmol} \mathrm{L}^{-1}$, at the end and beginning of the filtration test respectively; and $V_{t}$ and $V_{0}$ are the feed volumes, L, at the end and beginning of the filtration test respectively.

The fundamental performance with respect to $J_{\mathrm{W}}$ and $J_{\mathrm{DS}}$ of the virginal and modified membranes were initially evaluated using $1 \mathrm{~L}$ of deionized water as the feed solution and $1 \mathrm{~L}$ of $\mathrm{NaCl}$ 

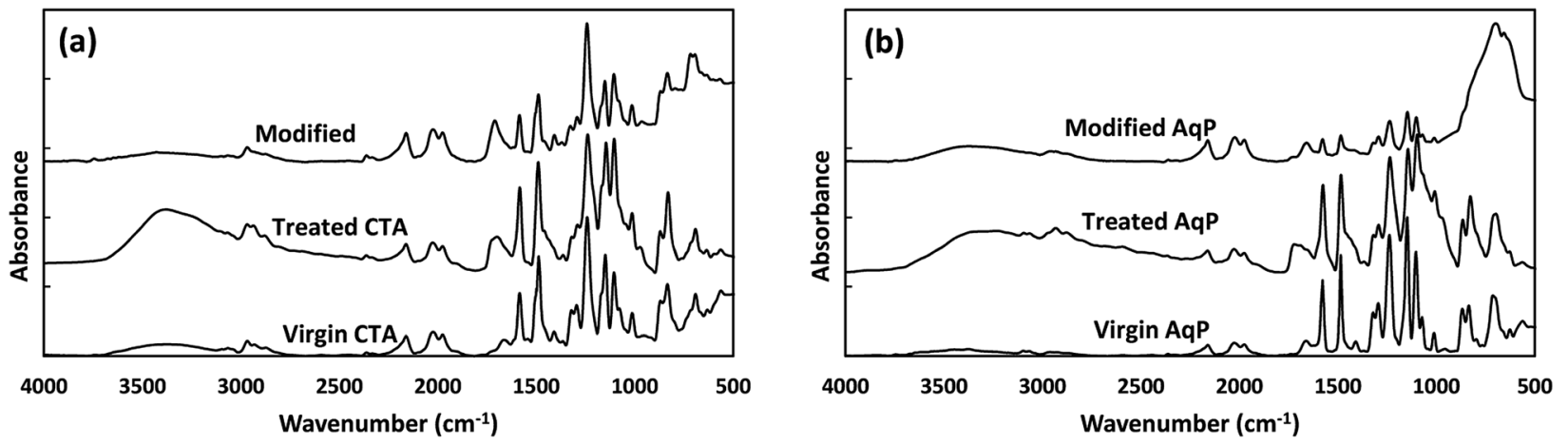

Fig. 2 FT-IR spectra of membrane active surfaces: (a) CTA membrane and (b) AqP membrane.

solution with a molar concentration of $1 \mathrm{moL} \mathrm{L}^{-1}$ as the draw solution. Both active layer-facing-feed solution (AL-FS) and active layer-facing-draw solution (AL-DS) orientations of the virgin membranes were tested, while the modified membranes were tested using only the AL-FS orientation. This is because the surface modification was performed particularly on the active surfaces. Two hours of batch filtration tests were performed, with the average flux reported in this study. The specific reverse diffusion rate, $\xi\left(\mathrm{mmoL} \mathrm{L}^{-1}\right.$ ), was employed to assess the membrane selectivity on the draw solute, which is determined as follows:

$$
\xi=\frac{J_{\mathrm{DS}}}{J_{\mathrm{W}}}
$$

In terms of the membrane antifouling performance, humic acid (Sigma-Aldrich, USA) was selected as a model of the organic foulants in the feed solution. Humic acid was dissolved at $15 \mathrm{mg} \mathrm{L}^{-1}$ in $1 \mathrm{~L}$ of salt solution composed of 0.5 $\mathrm{mmoL} \mathrm{L}^{-1} \mathrm{CaCl}_{2}$ and $1 \mathrm{mmoL} \mathrm{L}^{-1} \mathrm{NaHCO}_{3}$ to form an organic feed solution. Prior to the fouling experiment, a baseline measurement was conducted to present the flux lost caused by draw solution dilution over time. A feed solution composed of $7 \mathrm{mmoL} \mathrm{L}^{-1} \mathrm{NaCl}, 0.5 \mathrm{mmoL} \mathrm{L}^{-1} \mathrm{CaCl}_{2}$, and $1 \mathrm{mmoL} \mathrm{L}^{-1}$ $\mathrm{NaHCO}_{3}$, with equivalent conductivity to the organic feed solution, was used in the baseline test. Following the baseline measurement, four cycles of the fouling experiment were performed using the organic feed solution, with each cycle lasting for $12 \mathrm{~h}$. At the end of each cycle, the feed and draw solutions were replaced, thus the flux lost caused by membrane fouling could be identified via comparing the flux measured for each cycle with that of the baseline and former fouling cycles. $1 \mathrm{~L}$ of $1 \mathrm{moL} \mathrm{L}^{-1} \mathrm{NaCl}$ was used as the draw solution in the fouling experiment.

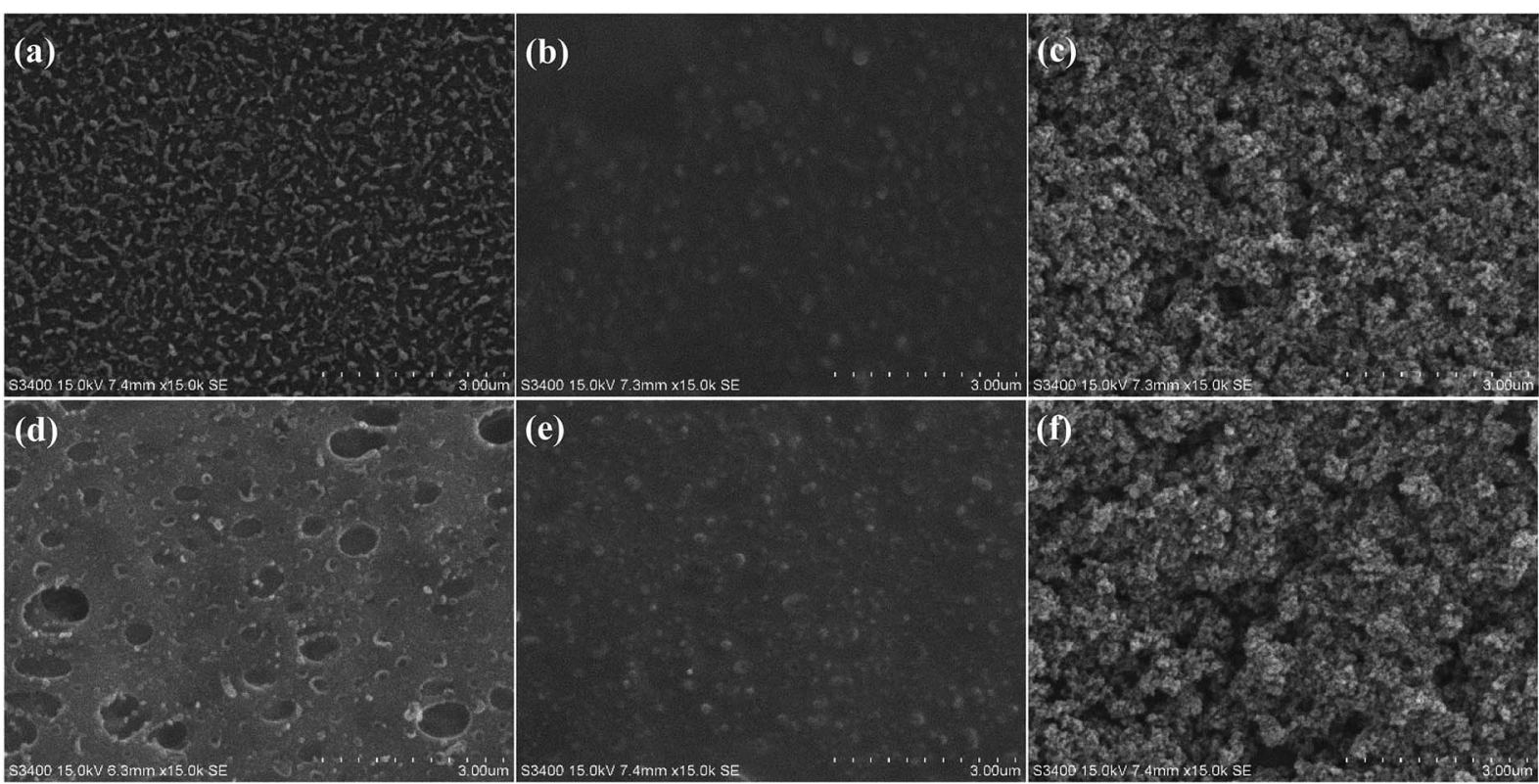

Fig. 3 SEM images of membrane active surfaces: (a) virgin CTA membrane, (b) treated CTA membrane, (c) modified CTA membrane, (d) virgin AqP membrane, (e) treated AqP membrane, and (f) modified AqP membrane. 

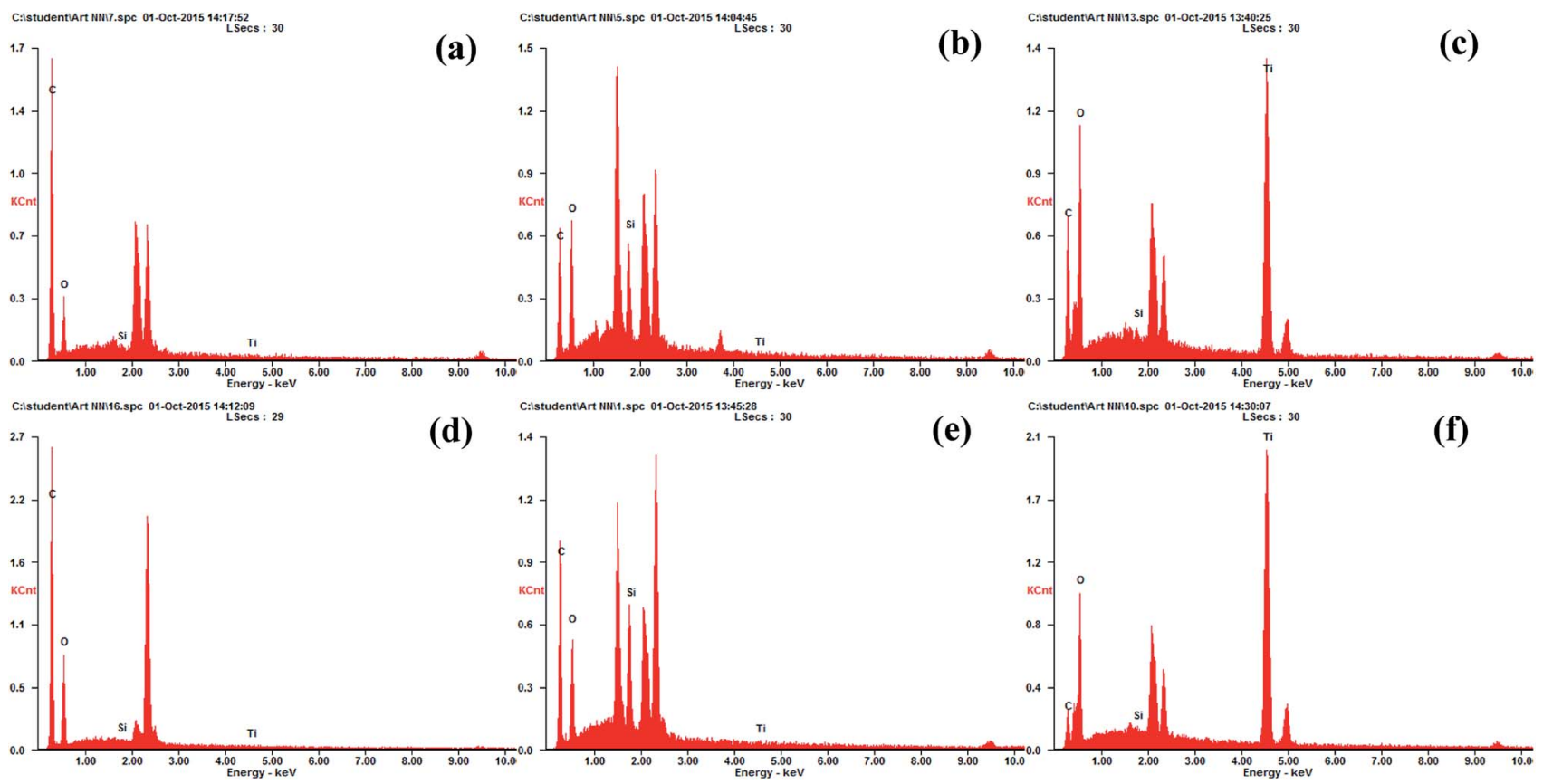

Fig. 4 EDX spectra of membrane active surfaces: (a) virgin CTA membrane, (b) treated CTA membrane, (c) modified CTA membrane, (d) virgin AqP membrane, (e) treated AqP membrane, and ( $f$ ) modified AqP membrane.

\section{Results and discussion}

\subsection{Effect of $\mathrm{TiO}_{2}$ coating on membrane surface characteristics}

3.1.1 FT-IR. FT-IR analysis was conducted to evaluate the variation in functional groups during surface modification. Fig. 2 shows the absorbances of virgin membranes, MEMOPMMA-Br treated (hereafter referred to as treated) membranes, and $\mathrm{TiO}_{2}$ modified membranes. For both virgin CTA and AqP membranes (Fig. 2(a) and (d)), absorbance peaks at wave numbers of $\sim 1100 \mathrm{~cm}^{-1}, \sim 1240 \mathrm{~cm}^{-1}$, and $\sim 1500 \mathrm{~cm}^{-1}$ were observed, which are representative of $\mathrm{C}-\mathrm{O}, \mathrm{C}-\mathrm{O}-\mathrm{C}$, and aromatic $\mathrm{C}=\mathrm{C}$ asymmetric stretching vibrations, respectively. ${ }^{30}$ The results are generally consistent with the specific FT-IR absorbance peaks for CTA and TFC membranes reported by $\mathrm{Xu}$ et $a l .^{38}$ After being treated by the MEMO-PMMA-Br monomers and hydrogenation, a broad peak at $\sim 3360 \mathrm{~cm}^{-1}$ appeared on the FT-IR spectra (Fig. 2(b) and (e)), which is potentially attributed to the reflectance of the $-\mathrm{Si}-\mathrm{OH}$ group..$^{36}$ Meanwhile, the absorbance at wave number $\sim 1700 \mathrm{~cm}^{-1}$ was significantly intensified, probably due to the increase of $\mathrm{C}=\mathrm{O}$ groups. On the other hand, absorbance of the $\mathrm{C}-\mathrm{Br}$ group was not observed, indicating a potential covalent linkage between the membrane surface and the MEMO-PMMA-Br chain through substitution of brominated hydrocarbon. These changes in FT-IR absorbance presented a successful grafting of the MEMO-PMMA-Br monomer chains onto the active surface of FO membranes. Deposition of $\mathrm{TiO}_{2}$ nanoparticles was subsequently carried out. The FT-IR spectra are shown in Fig. 2(c) and (f) for the $\mathrm{TiO}_{2}$ modified CTA and AqP membranes, respectively. Intense Ti-O bands were found at wave numbers ranging from 500 to $800 \mathrm{~cm}^{-1},{ }^{39}$ and a decrease at wave $\sim 3000 \mathrm{~cm}^{-1}$ was attributed to the silanization of $\mathrm{TiO}_{2}$ nanoparticles on the membrane active surfaces. ${ }^{27}$

3.1.2 SEM-EDX and AFM. To confirm that the $\mathrm{TiO}_{2}$ nanoparticles were successfully coated onto the active layers of CTA and AqP membranes, the active surfaces were characterized via SEM (Fig. 3) combined with EDX (Fig. 4). The SEM images illustrated the effective deposition of $\mathrm{TiO}_{2}$ nanoparticles on $\mathrm{FO}$ membranes after the modification procedure. $\mathrm{TiO}_{2}$

Table 1 Surface element coverages on the virgin, treated, and modified forward osmosis membranes

\begin{tabular}{|c|c|c|c|c|}
\hline & \multicolumn{4}{|c|}{ Surface element coverage, at $\%$} \\
\hline & Carbon (C) & Oxygen (O) & Silicon $(\mathrm{Si})$ & Titanium (Ti) \\
\hline Treated CTA membrane & $55.65 \pm 3.11$ & $36.72 \pm 2.95$ & $7.24 \pm 1.56$ & $0.39 \pm 0.21$ \\
\hline $\mathrm{TiO}_{2}$ modified CTA membrane & $16.66 \pm 7.60$ & $54.52 \pm 1.26$ & $0.53 \pm 0.24$ & $28.29 \pm 6.33$ \\
\hline Virgin AqP membrane & $67.77 \pm 1.29$ & $31.95 \pm 1.33$ & $0.15 \pm 0.05$ & $0.13 \pm 0.03$ \\
\hline
\end{tabular}


nanoparticles were uniformly scattered on both CTA and AqP membranes, turning the smooth active surfaces into ones covered by clusters and possessed porous structures. To quantitatively evaluate the coverage of titanium on both membranes, EDX spectra measurements were performed. Four major elements, carbon (C), oxygen (O), silicon (Si), and titanium (Ti), were identified from the EDX spectra. The increase of silicon coverage after spraying the MEMO-PMMA-Br reagent on the membrane surface showed good binding between the membrane surface and the monomer chains. The comparable coverage of silicon on the AqP and CTA membrane surfaces (Table 1) indicates the equivalent binding ability of the MEMOPMMA-Br monomer on both polyamide and cellulose triacetate active layers. The surface atomic percentages of titanium increased to approximately $30 \%$ after modification by $\mathrm{TiO}_{2}$ nanoparticles for both membranes. Meanwhile, the surface silicon coverage decreased, which was probably blocked by $\mathrm{TiO}_{2}$ nanoparticles, suggesting the bonding between $\mathrm{TiO}_{2}$ and MEMO-PMMA-Br monomer chains was through the silanols.

AFM was performed to visualize the variation of surface roughness before and after modification (Fig. 5). A significant increased surface roughness was observed in the modified membranes. This result generally supports the observations of the SEM images and indicates the successful surface coating of $\mathrm{TiO}_{2}$ on both FO membranes. Additional surface roughness information provided by a two-dimensional stylus profilometer supported the results obtained from AFM, with the average roughness of the CTA membrane increased from $554.84 \mathrm{~nm}$ to $622.51 \mathrm{~nm}$, and that for the AqP membrane increased from $303.23 \mathrm{~nm}$ to $440.64 \mathrm{~nm}$ (Fig. S2 $\dagger$ ). The changes in surface roughness are generally consistent with the observation by Nguyen et $_{\text {al. }}{ }^{29}$

3.1.3 Water contact angle. The water contact angles of the virgin, treated, and $\mathrm{TiO}_{2}$ modified $\mathrm{FO}$ membranes were
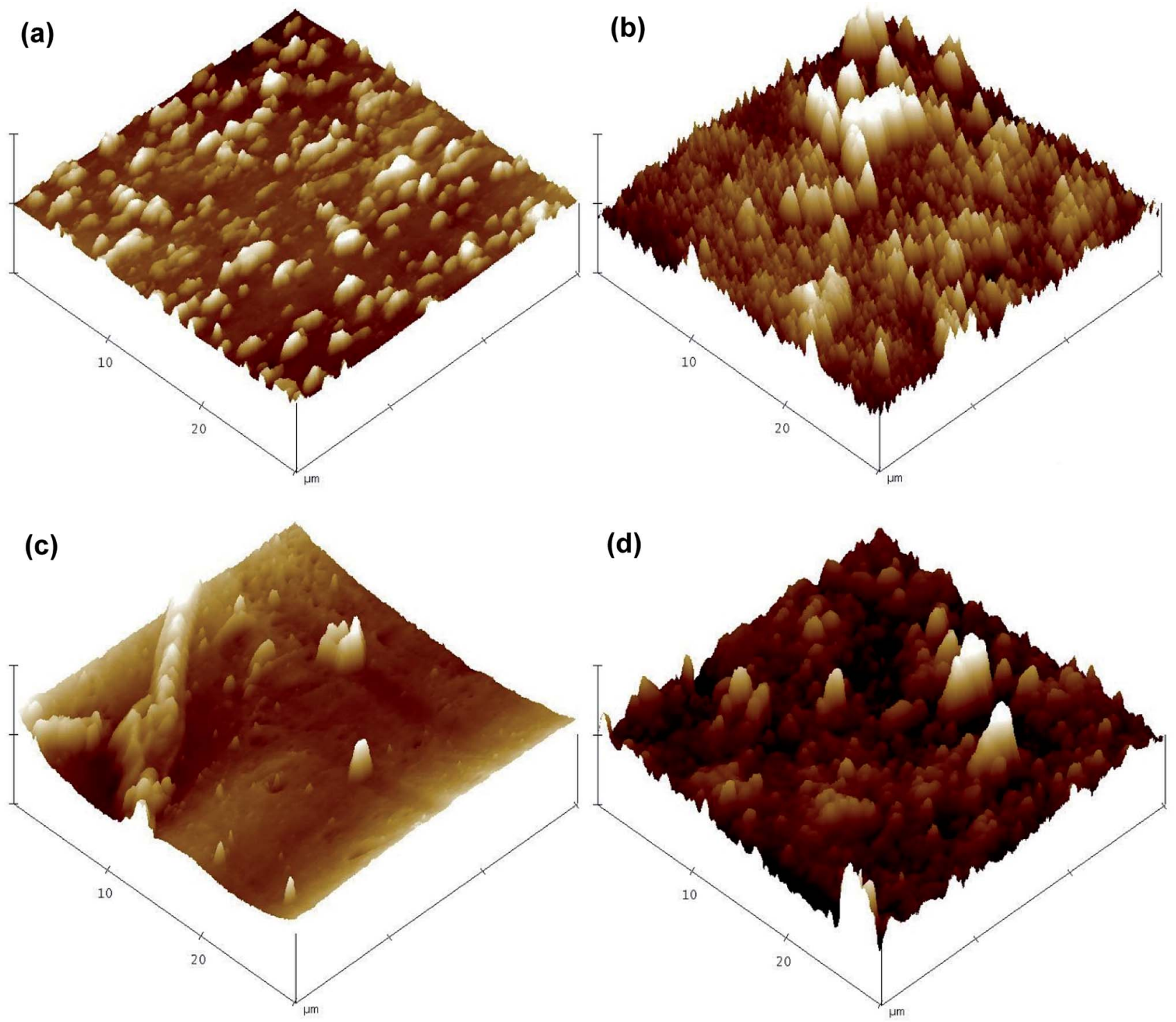

Fig. 5 AFM images of membrane active surfaces: (a) virgin CTA membrane, (b) modified CTA membrane, (c) virgin AqP membrane, and (d) modified AqP membrane. 
Table 2 Water contact angles of the virgin, treated, and modified forward osmosis membranes

Water contact angle $\left(^{\circ}\right)$
Virgin CTA membrane

Treated CTA membrane

$\mathrm{TiO}_{2}$ modified CTA

membrane

Virgin AqP membrane

Treated AqP membrane

$\mathrm{TiO}_{2}$ modified AqP membrane
$60.67 \pm 1.27$
$52.69 \pm 1.69$
39.6
$41.22 \pm 2.24$
$44.35 \pm 1.15$
60.59

measured to understand the effects of $\mathrm{TiO}_{2}$ surface modification on membrane hydrophilicity (Table 2). The water contact angles of the virgin CTA and AqP membranes were $60.67 \pm 1.27^{\circ}$ and $41.22 \pm 2.24^{\circ}$, respectively. As anticipated, there was a smaller contact angle for the AqP membrane than the CTA membrane, due to the improved surface hydrophilicity via embedding aquaporin molecules into the polyamide active surface. Binding with the MEMO-PMMA-Br monomer chains slightly increased the contact angle of the AqP membrane and decreased that of the CTA membrane. However, the variations on surface hydrophilicity caused by spraying the MEMOPMMA-Br reagent were insignificant.

The surface hydrophilicity of the CTA membrane was significantly enhanced by modification with $\mathrm{TiO}_{2}$ nanoparticles, with the average contact angle decreasing from $60.67^{\circ}$ to $39.60^{\circ}$. This conforms to the conclusion reported by $\mathrm{Yu}$ et $a .^{\mathbf{4 0}}$ that all freshly prepared porous $\mathrm{TiO}_{2}$ thin films using the sol-gel method possessed the surface contact angles of approximately $40^{\circ}$. In contrast, the average contact angle of the AqP membrane increased from $41.22^{\circ}$ to $60.59^{\circ}$, indicating an adverse effect on the hydrophilicity via surface modification with $\mathrm{TiO}_{2}$ nanoparticles. One possible reason may be that the coverage of MEMO-PMMA-Br monomers and $\mathrm{TiO}_{2}$ nanoparticles reduced the amount of the exposed aquaporin, which consequently weakened the surface hydrophilicity of the AqP membrane.

\subsection{Effect of $\mathrm{TiO}_{2}$ coating on membrane filtration} performance

The pure water flux for virgin and modified FO membranes was measured using $1 \mathrm{moL} \mathrm{L}^{-1} \mathrm{NaCl}$ as the draw solution, and the results are illustrated in Fig. 6. The AqP membrane exhibited a water flux of $10.92 \pm 0.72 \mathrm{~L} \mathrm{~m}^{-2} \mathrm{~h}^{-1}$ with AL-FS orientation and $11.07 \pm 0.38 \mathrm{~L} \mathrm{~m}^{-2} \mathrm{~h}^{-1}$ with AL-DS orientation, obviously higher than that of the CTA membrane of $7.04 \pm 1.30 \mathrm{~L} \mathrm{~m}^{-2} \mathrm{~h}^{-1}$ with AL-FS orientation and $10.94 \pm 0.34 \mathrm{~L} \mathrm{~m}^{-2} \mathrm{~h}^{-1}$ with AL-DS orientation. The water flux of modified FO membranes was measured with the AL-FS orientation. $73.4 \%$ and $13.6 \%$ of flux increments were obtained for CTA and AqP membranes after the surface modification, respectively. It is worth noting that the water flux of the CTA membrane was significantly improved by $\mathrm{TiO}_{2}$ nanoparticle modification, which is consistent with the improved surface hydrophilicity. Meanwhile, the reverse diffusion of salt for both orientations of virgin membranes and the AL-FS orientation of modified membranes were evaluated. The $\xi$ value for the modified AqP membrane $\left(0.20 \mathrm{mmoL} \mathrm{L}{ }^{-1}\right)$ was similar to that for the virgin membrane $\left(0.16 \mathrm{mmoL} \mathrm{L}^{-1}\right)$, whereas the $\xi$ value for the modified CTA membrane $(0.09$ mmoL $\left.\mathrm{L}^{-1}\right)$ was half of that for the virgin CTA membrane $(0.18$ mmoL $\mathrm{L}^{-1}$ ), presenting a better solute selectivity of the modified membrane. Although the flux performance of the commercial AqP membrane was much better than that of the CTA membrane due to the AqP's biomimicry molecular design, the performance of the modified CTA membrane became equivalent to or surpassed that of the AqP membrane in this study.

\subsection{Effect of $\mathrm{TiO}_{2}$ coating on membrane antifouling performance}

The development of membrane fouling was studied via conducting a series of fouling cycles for each membrane sheet and comparing the variation of flux patterns during the fouling cycles (Fig. 7). A baseline measurement was obtained prior to the fouling cycles, using $\mathrm{NaCl}$ to substitute the model foulants in the feed solution in order to maintain an equivalent initial
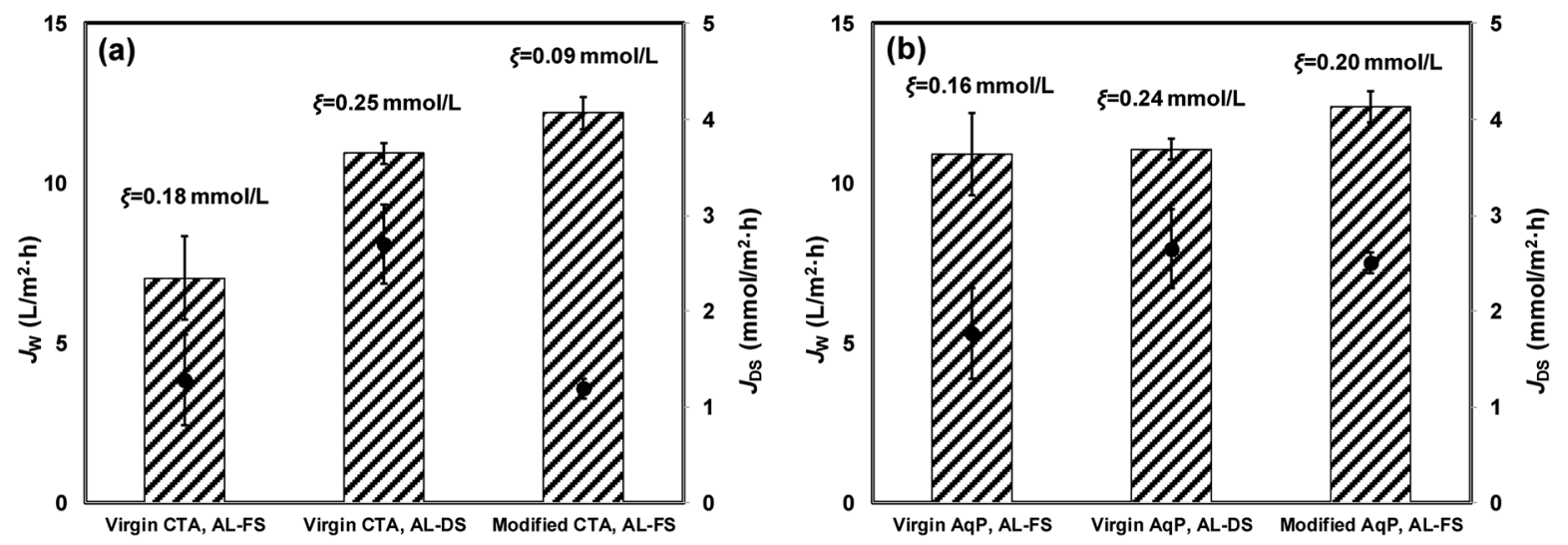

Fig. 6 Forward osmosis water flux, reverse solute flux, and specific reverse diffusion rate for (a) CTA membrane and (b) AqP membrane. The columns with error bars show the averages and standard deviations of water flux; the scatters with error bars show the averages and standard deviations of reverse salt flux; $\xi$ is the specific reverse diffusion rate. 

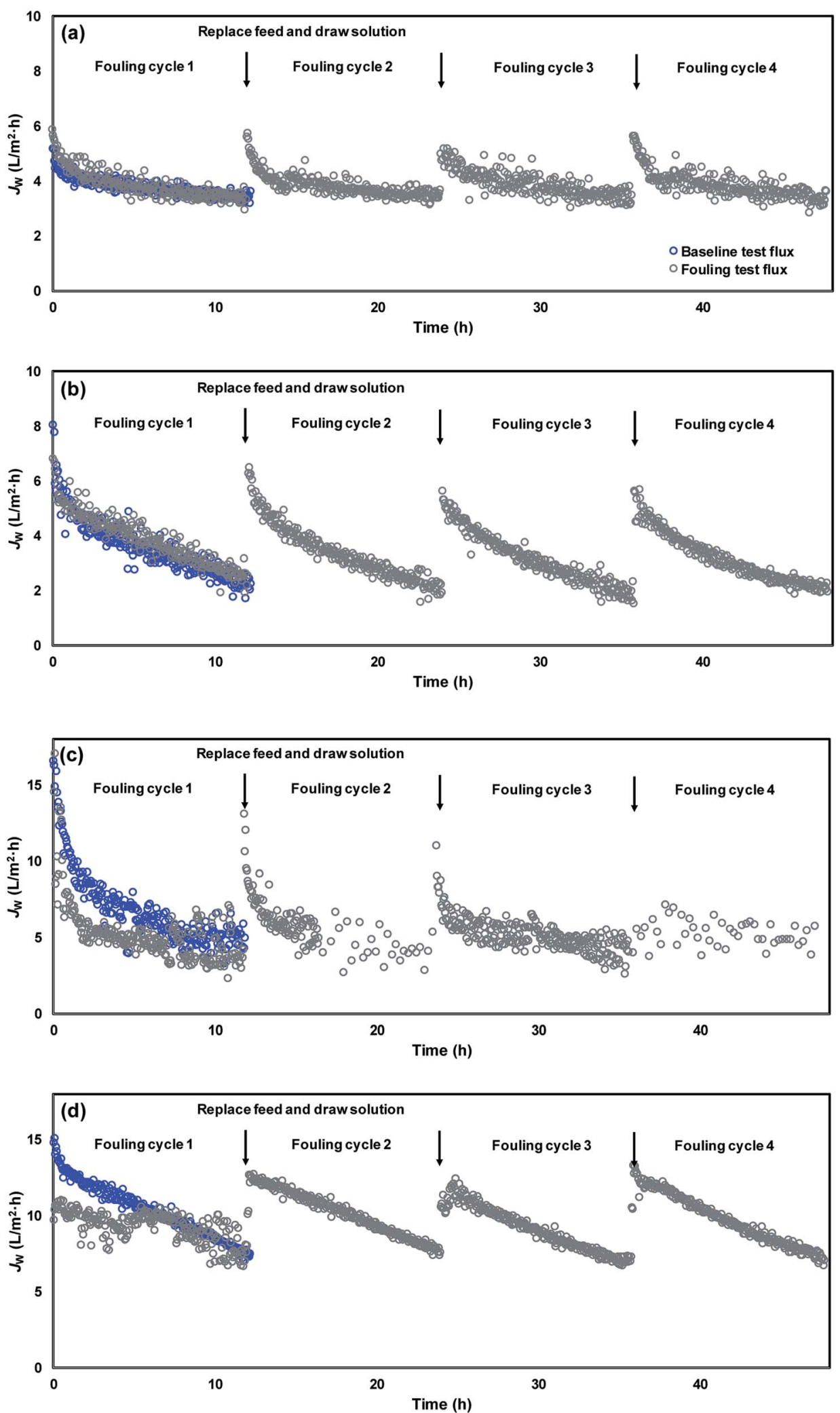

Fig. 7 Membrane flux variation during organic fouling tests for (a) virgin CTA membrane, (b) modified CTA membrane, (c) virgin AqP membrane, and (d) modified AqP membrane.

conductivity. Therefore, comparable water flux was expected between the baseline and the first fouling cycle at the beginning of the filtration test. The flux decline that occurred in the baseline measurement was due to the integrated effects of the dilution of draw solution and the concentration polarization. The difference observed between the first fouling cycle and the 
baseline can be attributed to the effect of membrane fouling. It can be seen in Fig. 7(a) and (b) that CTA membrane fouling was not observed in the first fouling cycles for either virgin or modified membranes. To zoom the difference on flux variation caused by membrane fouling, four cycles of fouling tests were continually conducted, with each test lasting for approximately $12 \mathrm{~h}$.

The variation of the average water flux for the initial $30 \mathrm{~min}$ of the baseline measurement and different fouling cycles is presented in Fig. 8. Although the initial flux of the modified membrane was always higher than that of the virgin membrane, there was no apparent decline of the initial water flux in each series observed. The strong antifouling property of the CTA membrane was indicated, which has been verified by several previous studies. ${ }^{\mathbf{1 1 , 4 1 , 4 2}}$ Nevertheless, since there was no significant membrane fouling observed even for the virgin CTA membrane, it is difficult to conclude whether the surface modification resulted in obvious enhancement of the antifouling performance of the membrane from the current data. Further studies with more fouling cycles may be needed to clarify this issue in the future.

In terms of the AqP membrane, slightly higher water flux was observed in the baseline for the modified AqP membrane than that for the virgin membrane, which was consistent with the results obtained in Section 3.2. Flux decline caused by membrane fouling was apparently observed in the first fouling cycle for the virgin AqP membrane (Fig. 7(c) and (d)). In addition, the membrane fouling developed dramatically in the following fouling cycles, with the initial water flux dropping from $13.6 \mathrm{~L} \mathrm{~m}^{-2} \mathrm{~h}^{-1}$ to $4.0 \mathrm{~L} \mathrm{~m}^{-2} \mathrm{~h}^{-1}$ (Fig. 8). This rapid fouling development in may be caused by the clogging of the functional water channels in aquaporins. Due to limited available information on the antifouling performance of commercial AqP membranes, this finding may be of great significance for the further improvement of the membrane material design.

In contrast, a constant initial water flux at approximately 12.0 $\mathrm{L} \mathrm{m}^{-2} \mathrm{~h}^{-1}$ was well maintained during the four fouling

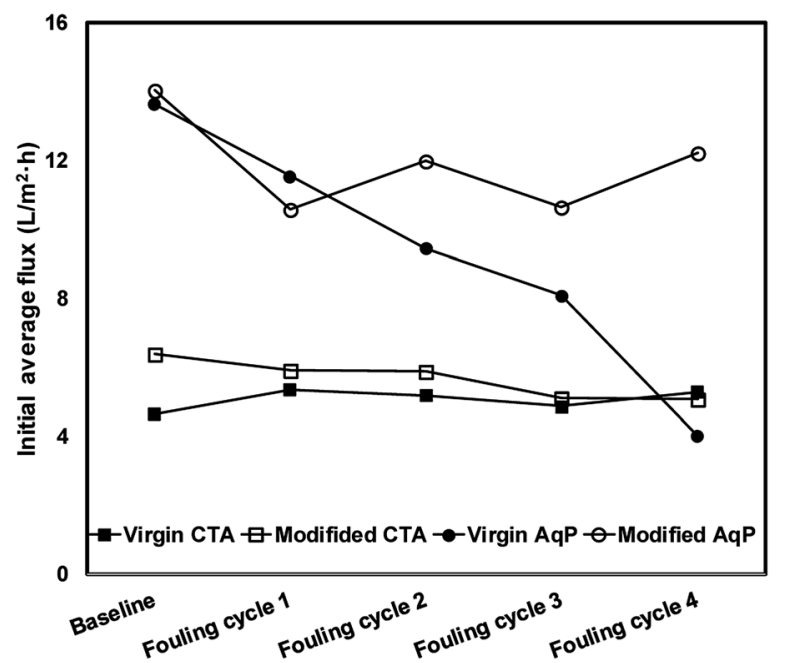

Fig. 8 The average water flux in the initial $30 \mathrm{~min}$ of the baseline and fouling cycle experiments. cycles for the modified AqP membrane, indicating the improved antifouling performance by surface modification in this study. It is worth mentioning that the low flux appeared at the beginning of the first fouling cycle may be caused by improper data collection which was rectified later in the experiment. Apart from the photodegradation of organic foulants which was catalyzed by $\mathrm{TiO}_{2}$ modified membrane interface, another possible mechanism is that the surface coverage by MEMOPMMA-Br monomer chains and $\mathrm{TiO}_{2}$ nanoparticles partially prevented the approach of organic foulants, obstructing them from blocking the functional water channels.

\subsection{Stability of the surface modified FO membranes}

According the previous reports, the stability of $\mathrm{TiO}_{2}$ modified membranes is usually a major concern for the application. ${ }^{27}$ The word "stability" here can be interpreted from two aspects, the integrity of the membrane structure and the adhesion of $\mathrm{TiO}_{2}$ to the membrane. In this study, the design of the monomer chain as a medium to graft the $\mathrm{TiO}_{2}$ nanoparticles to a polymer surface aimed to decrease the potential damage caused by oxidation of the membrane polymer. The stability of the reported modification method was indirectly reflected in the results of the membrane fouling examination (see Fig. 7(b) and (d)). After four cycles of fouling experiment, a dramatic flux increase was not observed for either modified membrane, which indicates that damage to the membrane materials caused by oxidation was limited. On the other hand, the enhanced antifouling performance was well maintained, revealing that the surface activity via modification remained effective. However, loss of $\mathrm{TiO}_{2}$ from the membrane surface was observed after several filtration cycles. Further efforts on extending the duration of $\mathrm{TiO}_{2}$ bonding and activity are needed.

\section{Conclusions}

$\mathrm{TiO}_{2}$ nanoparticles were successfully grafted onto commercial CTA and AqP FO membranes through a specially designed MEMO-PMMA-Br monomer chain. Titanium coverage of approximately $30 \%$ with a significant increase of the surface roughness was identified via surface observations using FT-IR, SEM-EDX and AFM. The surface hydrophilicity of the modified CTA membrane was significantly improved, with the average water contact angle decreasing from $60.67^{\circ}$ to $39.60^{\circ}$. A further test on the pure water flux strongly supported this observation: the water flux of the modified CTA membrane was increased by $73.4 \%$ compared to that without surface modification. On the other hand, the water contact angle of modified $\mathrm{AqP}$ membrane was found to increase from $41.22^{\circ}$ to $60.59^{\circ}$, it suggests that the water permeability was limited as the aquaporin was obstructed by the coverage of MEMO-PMMA-Br monomers and $\mathrm{TiO}_{2}$ nanoparticles. Consequently, only a slight improvement to the pure water flux of $13.6 \%$ was observed on modification. The improvement of the antifouling performance of the modified CTA membrane was insignificant, possibly because of the already excellent antifouling ability of the virgin CTA membrane. In contrast, the antifouling performance of the 
AqP membrane was greatly enhanced after surface modification, probably due to the integrated effects of foulant photodegradation (catalyzed by $\mathrm{TiO}_{2}$ nanoparticles at the interface) and the prevention of functional water channels being blocked by organic foulants due to $\mathrm{TiO}_{2}$ coverage. In addition, the stability of the coating method was demonstrated by the constant water flux and salt retention during the fouling tests.

\section{Conflicts of interest}

There are no conflicts of interests to declare.

\section{Acknowledgements}

This work was supported by the National Research Council of Thailand for Urban Water Reuse Project FY 2016, Ratchadapisek Sompoch Endowment Fund (2016) Chulalongkorn University (Grant No. CU-59-006-IC), the National Natural Science Foundation of China (Grant No. 51708408), and the KWEF-AIT Research Grant.

\section{References}

1 E. R. Cornelissen, D. Harmsen, K. F. de Korte, C. J. Ruiken, J. J. Qin, H. Oo and L. P. Wessels, J. Membr. Sci., 2008, 319, 158-168.

2 B. Mi and M. Elimelech, J. Membr. Sci., 2010, 348, 337-345.

3 S. Lee, C. Boo, M. Elimelech and S. Hong, J. Membr. Sci., 2010, 365, 34-39.

4 T. Y. Cath, S. Gormly, E. G. Beaudry, M. T. Flynn, V. D. Adams and A. E. Childress, J. Membr. Sci., 2005, 257, 85-98.

5 Y.-J. Choi, J.-S. Choi, H.-J. Oh, S. Lee, D. R. Yang and J. H. Kim, Desalination, 2009, 247, 239-246.

6 C. A. Nayak and N. K. Rastogi, Sep. Purif. Technol., 2010, 71, 144-151.

7 R. W. Holloway, A. E. Childress, K. E. Dennett and T. Y. Cath, Water Res., 2007, 41, 4005-4014.

8 A. Achilli, T. Y. Cath, E. A. Marchand and A. E. Childress, Desalination, 2009, 239, 10-21.

9 M. Xie, L. D. Nghiem, W. E. Price and M. Elimelech, Environ. Sci. Technol. Lett., 2014, 1, 191-195.

10 J. Zhang, Q. She, V. W. C. Chang, C. Y. Tang and R. D. Webster, Environ. Sci. Technol., 2014, 48, 3386-3394.

11 W. Xue, K. Yamamoto and T. Tobino, J. Membr. Sci., 2016, 499, 555-562.

12 J. R. McCutcheon, R. L. McGinnis and M. Elimelech, J. Membr. Sci., 2006, 278, 114-123.

13 R. Wang, L. Shi, C. Y. Tang, S. Chou, C. Qiu and A. G. Fane, J. Membr. Sci., 2010, 355, 158-167.

14 B. Kim, G. Gwak and S. Hong, Desalination, 2017, 421, 61-71.

15 S. Jamil, S. Jeong and S. Vigneswaran, Sep. Purif. Technol., 2016, 171, 182-190.

16 Q. Ge, M. Ling and T.-S. Chung, J. Membr. Sci., 2013, 442, 225-237.
17 W. Xue, K. Yamamoto, T. Tobino and C. Ratanatamskul, J. Membr. Sci., 2016, 515, 7-21.

18 J. Kim, J.-S. Chung, H. Kang, Y. A. Yu, W. J. Choi, H. J. Kim and J.-C. Lee, Macromol. Res., 2014, 22, 963-970.

19 M. Xie, J. Lee, L. D. Nghiem and M. Elimelech, J. Membr. Sci., 2015, 493, 748-754.

20 B. Meyer, Int. Biodeterior. Biodegrad., 2003, 51, 249-253.

21 B. Mi and M. Elimelech, J. Membr. Sci., 2008, 320, 292-302.

22 G. Kang and Y. Cao, Water Res., 2012, 46, 584-600.

23 K. P. Lee, T. C. Arnot and D. Mattia, J. Membr. Sci., 2011, 370, 1-22.

24 S. Lu, D. Wu, Q. Wang, J. Yan, A. G. Buekens and K. Cen, Chemosphere, 2011, 82, 1215-1224.

25 S. Yang, J.-S. Gu, H.-Y. Yu, J. Zhou, S.-F. Li, X.-M. Wu and L. Wang, Sep. Purif. Technol., 2011, 83, 157-165.

26 R. Mu, Z. Xu, L. Li, Y. Shao, H. Wan and S. Zheng, J. Hazard. Mater., 2010, 176, 495-502.

27 S. Leong, A. Razmjou, K. Wang, K. Hapgood, X. Zhang and H. Wang, J. Membr. Sci., 2014, 472, 167-184.

28 H. Song, J. Shao, Y. He, B. Liu and X. Zhong, J. Membr. Sci., 2012, 405-406, 48-56.

29 A. Nguyen, L. Zou and C. Priest, J. Membr. Sci., 2014, 454, 264-271.

30 M. Huang, Y. Chen, C.-H. Huang, P. Sun and J. Crittenden, Chem. Eng. J., 2015, 279, 904-911.

31 T. Cath, A. Childress and M. Elimelech, J. Membr. Sci., 2006, 281, 70-87.

32 K. Lutchmiah, A. R. D. Verliefde, K. Roest, L. C. Rietveld and E. R. Cornelissen, Water Res., 2014, 58, 179-197.

33 W. Xue, T. Tobino, F. Nakajima and K. Yamamoto, Water Res., 2015, 69, 120-130.

34 H. T. Madsen, N. Bajraktari, C. Hélix-Nielsen, B. Van der Bruggen and E. G. Søgaard, J. Membr. Sci., 2015, 476, 469474.

35 W. Ye, J. Lin, H. Tækker Madsen, E. Gydesen Søgaard, C. Hélix-Nielsen, P. Luis and B. Van der Bruggen, J. Membr. Sci., 2016, 498, 75-85.

36 R. Klaysri, S. Wichaidit, S. Piticharoenphun, O. Mekasuwandumrong and P. Praserthdam, Mater. Res. Bull., 2016, 83, 640-648.

37 P. Liu, H. Liu, G. Liu, K. Yao and W. Lv, Appl. Surf. Sci., 2012, 258, 9593-9598.

38 P. Xu, J. E. Drewes, T.-U. Kim, C. Bellona and G. Amy, J. Membr. Sci., 2006, 279, 165-175.

39 Q. Hu, E. Marand, S. Dhingra, D. Fritsch, J. Wen and G. Wilkes, J. Membr. Sci., 1997, 135, 65-79.

40 J. C. Yu, J. Yu, H. Y. Tang and L. Zhang, J. Mater. Chem., 2002, 12, 81-85.

41 R. Valladares Linares, V. Yangali-Quintanilla, Z. Li and G. Amy, J. Membr. Sci., 2012, 421-422, 217-224.

42 Z.-Y. Li, V. Yangali-Quintanilla, R. Valladares-Linares, Q. Li, T. Zhan and G. Amy, Water Res., 2012, 46, 195-204. 